\title{
Research Report \\ High Frequency Stimulation of the Ventrolateral and Mediodorsal Thalamic Nuclei: Differential Effects on Impulsive Action
}

\author{
Thibaut Sesia $a, b, d, e^{*}$ Koray Basar $a, c$ Rinske Vlamings $a, b, d, e$ Lee Wei Lim $a, b, d, e$ and Yasin Temel $a, b, d, e^{*}$ \\ a Department of Neuroscience, Medicine and Life Sciences, Maastricht University, Maastricht, The Netherlands; \\ b Department of Neurosurgery, University Hospital Maastricht, Maastricht, The Netherlands; \\ C Department of Psychiatry, Faculty of Medicine, Hacettepe University, Ankara, Turkey; \\ d Maastricht Institute for Neuromodulative Development (MIND) \\ e European Graduate School of Neuroscience (EURON)
}

\section{ARTICLE INFO}

\section{Article history:}

Received $06 / 06 / 2009$

Accepted 07 / 07 / 2009

\section{* Correspondence to:}

Yasin Temel,

Department of Neurosurgery,

University Hospital Maastricht,

P. Debyelaan 25, 6202

AZ Maastricht, The Netherlands

E-mail: y.temel@maastrichtuniversity.nl

\section{Keywords:}

Deep brain stimulation c-Fos, Mediodorsal thalamic nucleus, Premature responding, Reaction time task, Ventrolateral thalamic nucleus Rat

\section{ABSTRACT}

Ventrolateral thalamic nucleus stimulation is a frequently applied clinical procedure to control disabling tremor. Its motor effects are largely established now, but the cognitive effects are largely unknown and are currently under investigation. Here, we performed a detailed investigation of the effects of electrical stimulation at high frequency $(130 \mathrm{~Hz})$ and various amplitude $(0,1,3,30,150)$ of the $V L$ thalamic nucleus in a rat model on specific cognitive functions, using a reaction time task, and compared to DBS of the mediodorsal thalamic nucleus (MD) to evaluate the specificity of the site of stimulation. The involvement of the MD thalamic nucleus in cognitive functions has already been established. In addition, we mapped the effect of VL and MD thalamic nuclei DBS on the neuronal activity of the mPFC using c-Fos immunohistochemistry to investigate the neural pathways underlying the behavioural effects. Results show that DBS of the VL and MD thalamic nuclei had no significant effects on reaction time and motor time. DBS of the VL thalamic nucleus induced no significant effect on premature responding, whereas DBS of the MD thalamic nucleus increased this. In addition, MD and VL thalamic nuclei DBS significantly increased the number of c-Fos-immunoreactive cells in the prelimbic cortex as compared to control animals. In the infralimbic cortex, the number of c-Fos-immunoreactive cells was significantly higher following MD thalamic nucleus DBS as compared to the other groups. These findings support that the VL thalamic nuclei is not involved in impulsive action. However, DBS of the MD thalamic nucleus induced specific changes in cognitive parameters, which further establishes its involvement in behavioral functions.. This behavioral effect is mediated by a circuit involving specific prefrontal cortical areas. 


\section{Introduction}

Deep brain stimulation (DBS) of subcortical structures to control severely disabling neurologic and psychiatric disorders is a fast emerging area of clinical neuroscience (Wichmann and Delong, 2006). Thalamic DBS appeared at the end of the 80's for motor disorders, first in combination with thalamotomy on one side, and developed over decades to become a regular therapeutic approach (Benabid et al., 1987; Hubble et al., 1996; Lozano, 2000; Lyons and Pahwa, 2004). Thousands of patients worldwide have been implanted with stimulating electrodes and especially the thalamus has become a popular target in the management of pharmaco-resistant tremor (Schuurman et al., 2000).

Thalamic DBS is most effective when performed in the ventrolateral (VL) nucleus, which contains "tremor cells", and is nowadays predominantly used for essential tremor (Anderson et al., 2006; Kobayashi et al., 2003). (Alexander and Crutcher, 1990; Alexander et al., 1990; DeLong, 1990). Longterm results show that DBS of the VL thalamic nucleus induces a striking reduction of tremor, thereby confirming its powerful motor effects (Pahwa et al., 2006). From an anatomical point of view, the VL thalamic nucleus is part of the cortico-basal ganglia-thalamocortical motor circuit (Alexander and Crutcher, 1990; Alexander et al., 1990; DeLong, 1990). The cognitive effects of DBS of the VL thalamic nucleus, however, are less wellknown. In case series, authors have described subtle effects on memory functions (Loher et al., 2003; Troster et al., 1998), but its involvement in other cognitive domains still needs to be elucidated. Here, we performed a detailed investigation of the effects of electrical stimulation of the VL thalamic nucleus on cognitive functions in animal models. In the rat, the VL nucleus of the thalamus is considered as the paragon of a motor 'relay' nucleus of the thalamus; receiving input both from the cerebellum and the basal ganglia (Groenewegen et al., 1999). More specifically, we reasoned that the VL thalamic nucleus can play a role in cognitive function such as impulsive action because of its anatomical and functional relationship with the subthalamic nucleus (STN) via the output nuclei of the basal ganglia; STN DBS increased VL activity (Benazzouz et al., 2000). Additionally, STN DBS was reported to increase impulsive action in rats (Desbonnet et al., 2004). Therefore we hypothesized that VL thalamus DBS would decrease impulsive action.

Furthermore, we compared the behavioral effects of DBS of the VL thalamic nucleus with DBS of the mediodorsal thalamic nucleus (MD) to evaluate the specificity of the site of stimulation. MD nucleus of the thalamus is considered as a limbic nucleus given its driving inputs (e.g. amygdala, ventral pallidum and ventral tegmental aera) (Kuroda et al., 1998); its projection 'define' the prefrontal cortex (Uylings and van Eden, 1990). It has already been shown that the MD thalamic nucleus is involved in impulsive action. Selective neurotoxic lesions of this nucleus increased premature responding in rats (Chudasama and Muir, 2001). We expected a similar increase in premature responding with MD thalamic nucleus DBS at high frequency, since high frequency stimulation is thought to mimic a lesion (Benazzouz et al., 2004; Filali et al., 2004).

Impulsivity covers a wide range of behaviours. Varieties of impulsivity have been defined and are believed to have different underlying biological mechanisms (Evenden, 1999a;
Evenden, 1999b). Different aspects of impulsive behaviour have distinct models and evaluation paradigms. It is possible to segregate impulsive choice (i.e. impulsive decision-making) and impulsive action (i.e. failure in response inhibition) through specific behavioural tasks. Impulsive action is usually evaluated by reaction time paradigms (Winstanley et al., 2006). Therefore, we investigated the behavioural effects of VL and MD thalamic nuclei DBS in a reaction time (RT) task. Additionally, RT tasks are very suitable to be used in DBS experiments in animal models as it can be performed repeatedly, which allows testing the effects at various stimulation parameters. Finally, we functionally mapped the effect of VL and MD thalamic nuclei DBS on the MPFC using c-Fos immunohistochemistry to investigate the neural pathways underlying the behavioural effects.

\section{Results}

Table 1. Summary of the behavioral and immunohistochemical results presented as mean $\pm \mathrm{SEM}$.

\begin{tabular}{|cc|c|c|c|}
\hline & & Control & MD Thalamus & YL Thalamus \\
\hline Motor Time & Off & $0.86 \pm 0.025$ & $0.78 \pm 0.044$ & $0.72 \pm 0.065$ \\
(s) & 1 & & $0.78 \pm 0.050$ & $0.74 \pm 0.061$ \\
& 3 & & $0.79 \pm 0.055$ & $0.73 \pm 0.062$ \\
& 30 & & $0.01 \pm 0.062$ & $0.77 \pm 0.080$ \\
& 150 & & $0.79 \pm 0.053$ & $0.74 \pm 0.060$ \\
\hline Reaction Time & Off & $0.43 \pm 0.017$ & $0.44 \pm 0.037$ & $0.41 \pm 0.037$ \\
$(s)$ & 1 & & $0.44 \pm 0.034$ & $0.39 \pm 0.023$ \\
& 3 & & $0.43 \pm 0.024$ & $0.41 \pm 0.033$ \\
& 30 & & $0.43 \pm 0.032$ & $0.44 \pm 0.048$ \\
& 150 & & $0.46 \pm 0.033$ & $0.38 \pm 0.027$ \\
\hline Premature Responding Ratio & Off & $0.25 \pm 0.043$ & $0.25 \pm 0.056$ & $0.31 \pm 0.051$ \\
(ratio) & 1 & & $0.32 \pm 0.061$ & $0.21 \pm 0.034$ \\
& 3 & & $0.31 \pm 0.047$ & $0.18 \pm 0.036$ \\
& 30 & & $0.31 \pm 0.070$ & $0.23 \pm 0.060$ \\
& 150 & & $0.30 \pm 0.064$ & $0.21 \pm 0.046$ \\
\hline Infarlimbic Cortex Activation & \multirow{2}{*}{$4.4 \pm 2.11$} & $30.4 \pm 5.90$ & $14.0 \pm 1.68$ \\
(cFos-ir cell/40E5 pixles) & & & \\
Prelimbic Cortex Activation & $5.6 \pm 1.74$ & $35.5 \pm 5.49$ & $23.6 \pm 4.26$ \\
(cFos.ir cell/110E5 pixles) & & & \\
\hline
\end{tabular}
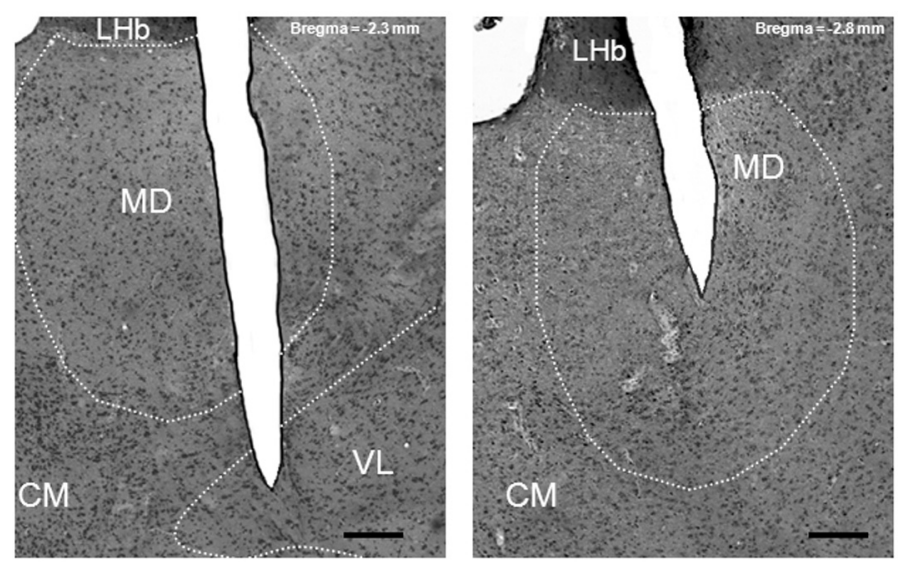

Fig. 1- Representative low-power photomicrograph of a $30 \mu \mathrm{m}$-thick frontal section from brains of rats with bilateral electrode implantation in the ventrolateral (VL) and mediodorsal (MD) thalamic nuclei. The tip of the electrodes are situated within the respective targets (scale bar $=200 \mu \mathrm{m}$ ). The surrounding structures are the lateral habenula (LHb) and the centromedian thalamic nucleus (CM). The white dotted lines show the boundaries of the structures and the dark lines delineate the boundaries of the electrodes trajectories.

All results are summarized in the table 1 as mean \pm SEM. 
Histological evaluation of electrode localization In 1 rat of $\mathrm{VL}$ thalamus group the electrodes were misplaced and 2 rats targeted in the VL thalamus and 3 rats targeted in the MD thalamus removed their electrode construction during the experiment. These rats were excluded from analysis. Electrode tips were located at the level of the VL thalamus in 5 rats and in the MD thalamus in 7 rats of group $C$. The localization of the electrode tip in the VL and MD thalamic nuclei are illustrated in Figure 1.

No histological damage was observed except for the electrode trajectory, suggesting that repeated stimulation with the current stimulation settings did not cause observable tissue damage.

Evaluation of the number of c-Fos-ir cells Since we performed bilateral stimulation and found no left/right difference in the number of c-Fos-ir cells, data from the left and right hemispheres were pooled (data not shown).Thalamic stimulation significantly increased the number of c-Fos-ir cells in the infralimbic cortex (F2,67=7.537, $p=0.001$; Figs.3 and 4), post-hoc analysis revealed that the MD thalamus DBS significantly increased the number of cFos-ir cells as compared with VL thalamus DBS and control groups. Furthermore, thalamic DBS resulted in significantly higher number of c-Fos-ir cells in the prelimbic cortex $(F 2,71=8.711, p<0.001$; Figs.3 and 4), post-hoc analysis showed significant increases of cFos-ir cells in both VL and MD thalamus DBS group as compared to control.

\section{Discussion}

Ventrolateral thalamic nucleus stimulation is a frequently applied clinical procedure to control disabling tremor Its motor effects are largely established now, but the cognitive effects are largely unknown and are currently under investigation. In this respect, utilizing animal models, we have found that no effect on PR or RT in a RT task. Additionally, MD thalamus stimulation modified the PR pattern by increasing this ratio at high frequency and low amplitude.

The histology revealed that thalamic DBS increased the number of cFos-ir cells in the infralimbic area of the MPFC in case of the MD thalamus DBS and in the prelimbic area with both sites of stimulation.

These findings provide further in vivo evidence for a link between the basal ganglia and VL thalamic nucleus. STN DBS in animal was reported to increase PR and to increase VL activity (Benazzouz et al., 2000; Desbonnet et al., 2004); but it seems that the information related to this effect does not pass by the VL thalamus since we observed no alteration of the PR pattern, irrespectively of the parameter of stimulation. Thus, the VL thalamic nucleus has to be seen as a motor nucleus of the thalamus which shares reciprocal connection with the somatosensory and frontal cortices: the latter areas were reported to send afferent connections to the MPFC, especially to the more dorsal part of the prefrontal cortex (Groenewegen and Witter, 2004; Heidbreder and Groenewegen, 2003). In line with this, we have found that DBS of the VL thalamic nucleus increased the number of c-Fos-ir in the prelimbic cortex.

Interestingly, in a clinical study lesions of this area reducedsome of the core symptoms of patients with Tourette's Syndrome, a disorder characterized by a deficit in impulsecontrol mechanisms (Babel et al., 2001). This suggests that ab-
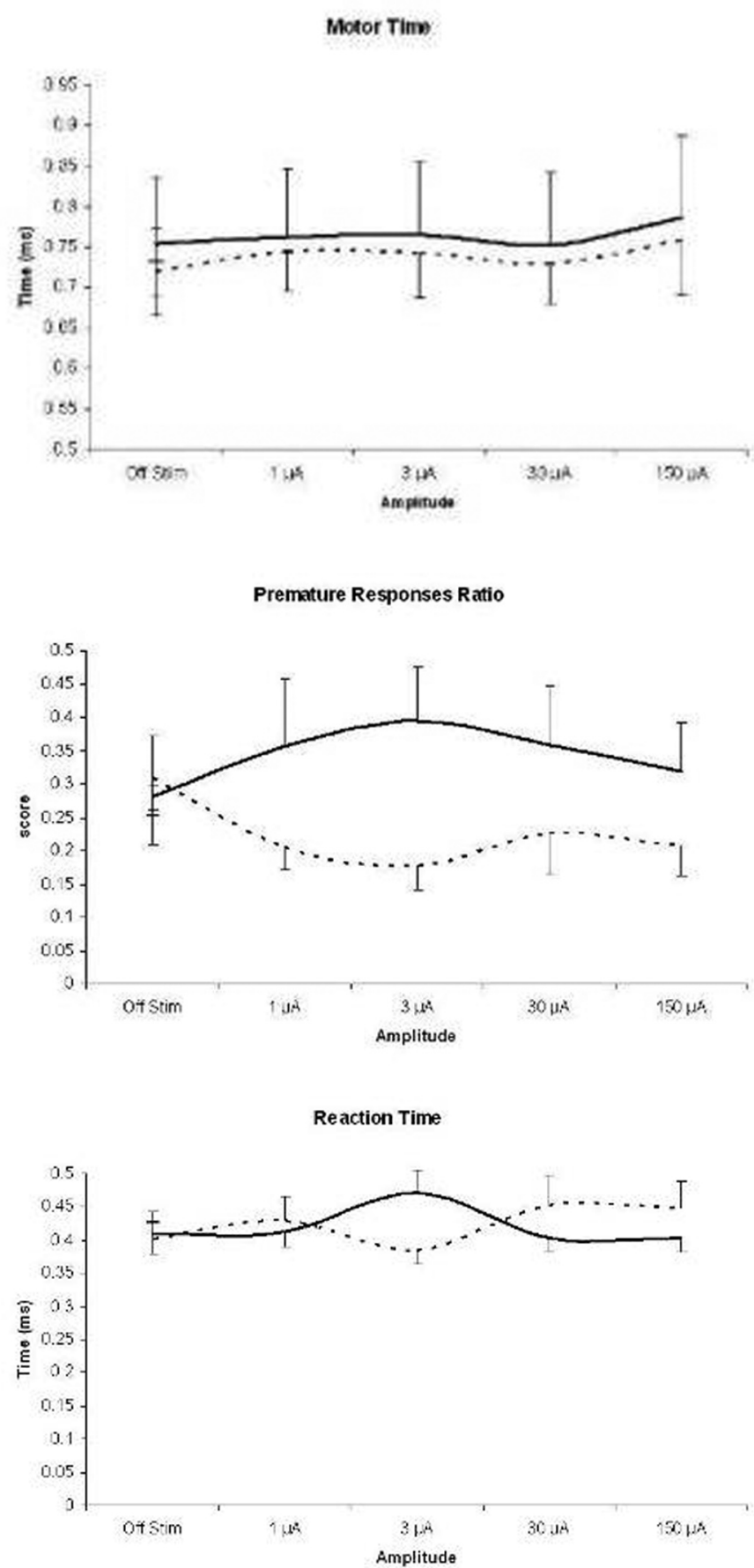

Fig. 2- Behavioural result of rats subjected to different stimulation amplitudes at bilateral high frequency stimulation at either MD (straight line) or VL (dotted line); score of non implanted animal (underscore). Data are shown as mean \pm SEM. Note the opposite effect of DBS of the MD and VL thalamic nuclei on premature responding. + indicates significant difference as compared to off stimulation within groups, * indicates significant difference between groups; $p$ $<0.05$.

lation of this area has a beneficial effect on impulsivity-related behaviours. This observations of Tourette's syndrome patients raise the question to which extent the VL thalamic nucleus can serve as a target for DBS in impulsivityrelated disorders. In our study, we have found no explanation for this beneficial 


\section{Cell Activation}

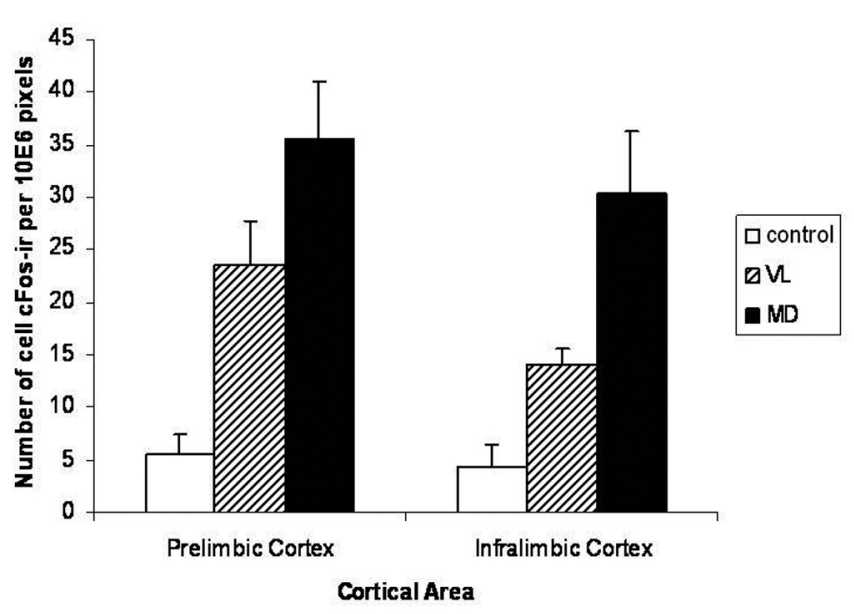

Fig. 3- Grouped data of c-Fos-ir cells per 105 pixels. Data represents means \pm S.E.M. of control animals, animals with VL thalamic nucleus DBS, and animals with MD thalamic nucleus DBS. Abbreviations: PrL: prelimbic cortex; IL: infralimbic cortex.* indicates significant difference between groups $(* \mathrm{p}<0.05)$.

effect, which could be due to the fact that we have used naïve rats and used DBS instead of lesions. We performed electrical stimulation of the MD thalamic nucleus which neighbors the $\mathrm{VL}$ thalamic nucleus. The MD thalamic nucleus is part of the cortico-basal ganglia-thalamocortical limbic circuit (Alexander et al., 1990).

High frequency stimulation of the MD thalamic nucleus induced a significant increase in PR. This confirms that the stimulation effects of $V L$ and $M D$ thalamic nuclei on $P R$ are region-specific. Increase in PR at baseline evaluation and during unpredictable intertrial interval while performing a similar RT task has also been reported with experimental lesions of the MD thalamic nucleus in rats (Chudasama and Muir, 2001). This similarity in behavioral performance supports the hypothesis that stimulation at high frequency can mimic a lesion-effect.

Furthermore, Chudasama et al. provided evidence that the MD thalamic nucleus and prelimbic cortex lesions induced different executive and attentional impairments, despite their direct and reciprocal connections (Chudasama and Muir, 2001; Groenewegen and Witter, 2004; Heidbreder and Groenewegen, 2003; Vertes, 2004; Vertes, 2006). In addition, they have demonstrated the functional differences of the subterritories of the $\mathrm{MPFC}$, which are all interconnected with the MD thalamic nucleus (Chudasama et al., 2003; Groenewegen, 1988; Ray and Price, 1992). The projection of the MD to the rat prefrontal cortex (PFC) is considered to define the rat PFC (Uylings and van Eden, 1990). More recently the concept of the ventral and dorsal $\mathrm{mPFC}$ has risen and both have strong connections with the MD thalamic nucleus.

Our c-Fos immunohistochemistry results are in accordance with this concept. Electrical stimulation of the MD thalamic nucleus significantly increased the number of c-Fosir cells in the prelimbic and infralimbic cortices suggesting an overall neuronal activation of these regions. Another important difference is that the MD and MPFC have extensive connections with the ventral striatum (i.e. the nucleus accum- bens) and connected to the STN via the ventral pallidum (e.g. output nucleus of the nucleus accumbens) (Groenewegen and Berendse, 1990; Groenewegen and Witter, 2004; Vertes, 2004). Both of these structure have been shown to be involved in impulsive processes by lesion, imaging or electrophysiological studies (Baunez and Robbins, 1997; Dalley et al., 2007; Desbonnet et al., 2004). It is interesting to notice that a larger activation of the MPFC (i.e. cFos-ir cells ratio) was associated with alteration of the PR pattern. Altogether the results support the current view of the MD and VL thalamic nuclei structures, using a new in vivo electrophysiological tool (animal DBS)

The underlying mechanism of electrical brain stimulation is being extensively investigated. Some authors argue a local inhibitory effect (Filali et al., 2004) and others a more complex effect of stimulation: local inhibition of the soma and generation of action potentials of the surrounding axons (Mclntyre et al., 2004a; Mclntyre et al., 2004b; Mclntyre et al., 2006), thereby superimposing a rhythmic output of the stimulated nucleus (Meissner et al., 2005). In addition, more and more emphasis is being put on the combination of orthodro-
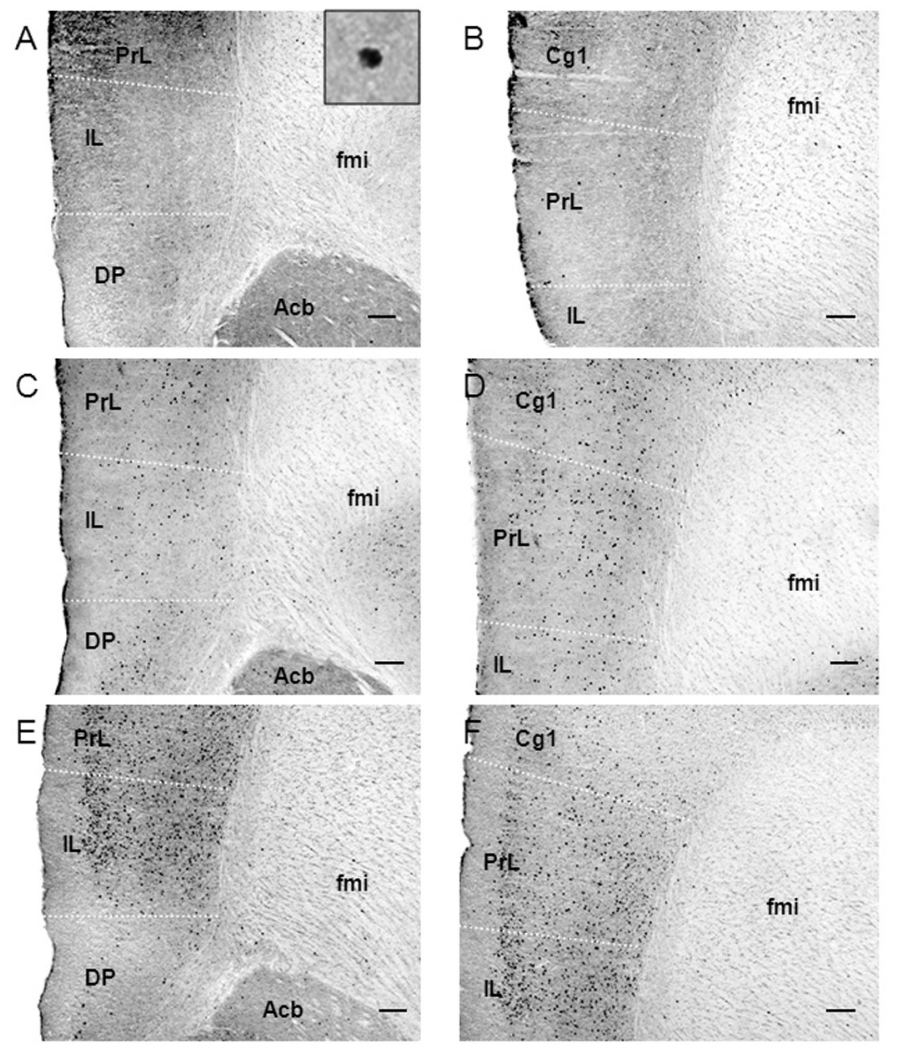

Fig. 4- Representative low-power photomicrographs of a $30 \mu \mathrm{m}$-thick frontal section from the brain of a control rat ( $A$ and $B$ ), a rat subjected to stereotactic implantation of concentric bipolar electrode to stimulate the VL thalamic nucleus ( $C$ and $D)$ and the MD thalamic nucleus ( $E$ and $F$ ). A, C, and $E$ show coronal sections taken at the level of the IL (Infralimbic cortex) and B, D, and $F$ show coronal sections taken at the level of PrL (Prelimbic cortex). The small dark dots represent c-Fos-ir cells. The inset in A shows a representative high-power photomicrograph of a c-Fos-ir cell. Scale bar $=300 \mu \mathrm{m}$. Abbreviations; fmi=forceps minor corpus callosum, $\mathrm{Cg} 1=$ Cingulate cortex area 1, DP=dorsopeduncular cortex. 
mic and antidromic action of electrical stimulation.

In this respect, it was recently shown that the electrophysiological effects of nucleus accumbens stimulation on the PFC is likely to be antidromic (McCracken and Grace, 2007). Parallel to the local mechanisms, investigations now focus on the network effect of DBS on the directly connected target nuclei (i.e. monosynaptic effects) (Tai et al., 2003) and indirectly connected target nuclei (i.e. polysynaptic effects) (Temel et al., 2007). Altogether, these concepts may help us to understand our results with respect to the mechanism of electrical stimulation. The MD thalamic nucleus is projecting glutamatergic to layers III and VI of the entire MPFC but also reciprocally to the non-pyramidal cell, which are GABA-ergic (Gabbott et al., 2005; Kuroda et al., 1998). It receives inputs from layer IV and V (Groenewegen, 1988). Our immunohistochemical evaluations revealed that the infralimbic and prefrontal cortices showed an overall activation, suggesting both orthodromic and antidromic current effect. Electrical stimulation of the MD thalamic nucleus may activate the pyramidal cells, whereby the activated non-pyramidal cells will inhibit them; thus reducing the input to the ventral striatum. This hypothesis would be in line with some imaging studies, which have suggested that hypo-activation of the PFC and ventral striatum were associated with impulsiveness (Rubia et al., 2005; Smith et al., 2006). Nevertheless, we cannot exclude a more direct effect through the amygdaloid complex (Groenewegen and Witter, 2004) or the shell subterritories of the nucleus accumbens (Vertes, 2004) (i.e. via the prelimbic and infralimbic cortices), since the MD thalamic nucleus is connected with these regions as well.

In conclusion, our study provided evidence against our hypothesis that the VL thalamic nucleus could be involved in impulsive action processes. Electrical stimulation of the MD thalamic nucleus had a strong effect on PR, Moreover, DBS appears to be a relevant translational tool that allows studying clinical questions with a mechanistic approach in the preclinical area: it allows us to study electrophysiological and behavioural phenomena beyond the direct anatomical connections.

\section{Experimental procedure Subjects}

All subjects were Lewis male rats ( $n=30,12$ weeks old, bred and housed at the Central Animal Facility of Maastricht University, Maastricht, The Netherlands), with an average body weight of $300 \mathrm{~g}$. Animals were housed individually in standard Makrolon $^{\mathrm{TM}}$ cages on sawdust bedding in an air-conditioned room (about $20^{\circ} \mathrm{C}$ ) under a 12/12-h reversed light/dark cycle (lights on from 18:00 to 6:00 h). All animals had free access to food and water. The rats were tested 5 days per week. During this time, the rats were given 10-12 g standard laboratory chow (Hopefarms, Woerden, The Netherlands) per day. On 2 days per week (weekend), they were left to rest and were fed ad libitum. This schedule reduced their weight to approximately $90 \%$ of their free feeding weight during the week. All experiments were approved by the Animal Experiments and Ethics Committee of Maastricht University.

\section{Behavioral evaluations}

This above mentioned task has been previously described (Temel et al., 2005b). In summary, rats were tested in Skinner chambers (inner dimensions: $40 \times 30 \times 33 \mathrm{~cm}$ ) equipped with two retractable levers with cue lights just above the levers. A food magazine ( $5 \times 5$ and $2.5 \mathrm{~cm}$ above the grid floor) was positioned equidistant between the two levers and could be accessed by pushing a hinged panel. The levers ( $4 \mathrm{~cm}$ wide) projected $2 \mathrm{~cm}$ into the conditioning chamber and were located 6 $\mathrm{cm}$ from both sides of the food tray and $12 \mathrm{~cm}$ above the grid floor. A light and a loudspeaker were fixed in the ceiling of the conditioning chamber. The test procedure was controlled by a personal computer and the data stored on disk at the end of a session (time precision $=1 \mathrm{~ms}$ ). During a trial, the rat must insert its nose into the central panel until a tone sounds. A high tone $(10 \mathrm{kHz} ; 80 \mathrm{~dB})$ required depression of the left lever and a low tone $(2.5 \mathrm{kHz} ; 80 \mathrm{~dB})$ the right lever. A variable period (randomly chosen from 0.6 to $1.5 \mathrm{~s}$, steps of $0.1 \mathrm{~s}$ ) between nose insertion and tone was termed the hold duration. When a rat did not hold the panel for the entire hold duration, the same interval was repeated when the panel was next pushed. Depression of the lever resulted in food reward in only $50 \%$ of cases to increase the enthusiasm of the animals for the task (Blokland, 1998). The reinforcement was given independently of the reaction time. Ten seconds elapsed between each trial. Each session lasted $30 \mathrm{~min}$ or until the completion of 60 trials.

To evaluate the behavioural performances prior to surgery, the rats underwent approximately two weeks of training until the mean reaction time of performances was stabilized. The parameters of the task used to investigate the response of the rats have been previously described and performed in our laboratory (Blokland, 1998).

Reaction time (RT): the mean latency between the onset of the tone and the release of the magazine panel was taken as the RT. It is generally accepted that response latencies shorter than $100 \mathrm{~ms}$ are unlikely to be true reaction times but should be considered as non-valid responses. On the other hand, response latencies longer than 1500 ms should not be considered as a task-related reaction time.

Motor time (MT): the mean latency between the release of the magazine panel and the lever press was taken as the motor time. It was assumed that motor times longer than $2 \mathrm{~s}$ did not reflect 'true' motor time.Premature responses ratio (PR): the total number of times the rat released the magazine panel before the hold duration had elapsed divided by the number of total trials. It should be noted that after a premature response the rats had to start the same trial again by pushing the hinged panel. In case of a premature response, the response lever was not inserted into the chamber.

\section{Surgical procedure}

Rats were randomly assigned to one of the following group: control (no surgery), bilateral electrode implantation in the VL thalamic nuclei, and bilateral electrode implantation in the MD thalamic nuclei. Animals were anaesthetized throughout the entire procedure using a combination of ketamine (90 $\mathrm{mg} / \mathrm{kg}$, s.c.) and xylazine (10 mg/kg, s.c.). Rats were placed in a stereotaxic apparatus (Stoelting, USA; model 51653). Two burr holes were made in the skull immediately above the targets to allow for insertion of electrodes. The stereotaxic coordinates from the bregma were for the MD thalamic nucleus $(a / p=-2.8$ $\mathrm{mm}, \mathrm{l} / \mathrm{v}= \pm 0.6 \mathrm{~mm}, \mathrm{~d} / \mathrm{v}=-5.0 \mathrm{~mm}$ ) and for the VL thalamic nucleus $(\mathrm{a} / \mathrm{p}=-2.3 \mathrm{~mm}, \mathrm{l} / \mathrm{v}= \pm 1.5 \mathrm{~mm}, \mathrm{~d} / \mathrm{v}=-6.5 \mathrm{~mm})$. A con- 
struction of two stainless steel electrodes (Technomed, The Netherlands), both concentric and bipolar, with a tip diameter of $50 \mu \mathrm{m}$ and a shaft diameter of $350 \mu \mathrm{m}$, was employed in this experiment. The electrodes were fixed in position using dental cement (Heraeus Kulzer, Germany). This technique of DBS in rodent has been described previously (Temel et al., 2007).

\section{Deep brain stimulation}

The stimulus was delivered using a World Precision Instrument (WPI, Berlin, Germany) accupulser (A310) and a stimulus isolator (A360). Real time verification of the parameters applied during stimulation was obtained for both electrodes of the bilateral construction, using a digital oscilloscope (Agilent Technologies, Agilent 54622D oscilloscope, The Netherlands). Stimulation started few minutes before the test and lasted for the duration of each session. Animals were tested once a day according to a latin-square schedule (see below) and had at least a 12-h off stimulation period before the next session started.

\section{Experimental procedures}

Rats were left for 1 week to recover from surgery after which they were trained in the Skinner boxes (modified to allow for the attachment of a stimulation cable), until behavioural parameters were stabilized. Following this, the average values of 2 sessions were calculated for each of the behavioural parameters mentioned above. This was done for various stimulation conditions:

1. With attachment of stimulation cable but without stimulation (off),

2. With stimulation at various amplitudes $(1,3,30,150$ $\mu \mathrm{A}), 60 \mu \mathrm{s}$ pulse width, and high frequency stimulation (HFS) at $130 \mathrm{~Hz}$.

3. With stimulation at various amplitudes $(1,3,30,150$ $\mu A), 60 \mu$ s pulse width, and low frequency stimulation (LFS) at $30 \mathrm{~Hz}$.

The order of the various amplitudes was randomized using a Latin square design in which each condition was repeated two times. Stimulation was performed with 150, 30, and $3 \mu \mathrm{A}$ to investigate the effect of a relatively high, medium and low current density with respect to the human condition. The frequency applied was low $(30 \mathrm{~Hz})$ and high $(130 \mathrm{~Hz})$ in order to determine whether changes were dependent on the pulse frequency. After completing the RT task, the rats were left for recovery for a week.

\section{Histological processing}

After a week of break, rats were stimulated for $30 \mathrm{~min}$ at $130 \mathrm{~Hz}$ and $30 \mu \mathrm{A}$ then for an hour at rest; rats were perfused transcardially with Tyrode $(0.1 \mathrm{M})$ and fixative containing $4 \%$ paraformaldehyde, $15 \%$ picric acid and $0.05 \%$ glutaraldehyde in $0.1 \mathrm{M}$ phosphate buffer ( $\mathrm{pH}$ 7.6). Brains were removed and postfixed for $2 \mathrm{~h}$ followed by overnight immersion in 10\% sucrose at $4{ }^{\circ} \mathrm{C}$. The solution of sucrose was increased to 20 $\%$ and finally to $30 \%$ each time the brains reached the bottom. Brain tissue was then quickly frozen with $\mathrm{CO} 2$ and stored at $-80^{\circ} \mathrm{C}$. Subsequently, the prefrontal regions of the brains were cut serially on a cryostat into $30 \mu \mathrm{m}$ frontal sections and again stored at $-80{ }^{\circ} \mathrm{C}$. C-Fos immunohistochemistry was carried out by using an anti-c-Fos rabbit polyclonal antibody
(Santa Cruz Biotechnology Inc., Santa Cruz, CA, USA) (diluted $1: 20000$ in $0.1 \%$ Bovine Serum Albumin and Tris Buffered Solution-Triton (TBS-T) solution). After overnight incubation at room temperature on a constant shaker, sections were rinsed in three steps with TBS-T, Tris Buffered Solution (TBS), and TBST and incubated with the secondary antibody (diluted 1: 400 in biotinylated donkey anti-rabbit; Jackson Immunoresearch Laboratories Inc., Westgrove, USA) for ninety minutes. Subsequently, the sections were incubated with an avidin-biotinperoxidase complex (diluted 1:800, Elite ABC-kit, Vectastatin; Burlingame, USA) for two hours. In between steps, sections were washed with TBS and TBS-T. To visualize the immune complex of Horse Radish Peroxide reaction product, sections were incubated with 3,3'-diaminobenzidine tetrahydrochloride (DAB)/ Nickel Chloride ( $\mathrm{NiCl} 2)$ solution ( $5 \mathrm{ml}$ DAB solution, $5 \mathrm{ml}$ Tris/ $\mathrm{HCl}, 50 \mu \mathrm{l} \mathrm{NiCL2}$, and $3.35 \mu$ l hydrogen peroxide). This reaction was stopped after 10 minutes by rinsing thoroughly all the sections with TBS. All sections were then mounted on gelatin-coated slides. After dehydrating, all sections were cover-slipped with Pertex (HistolabProducts ab, Goteborg, Sweden). Additionally, one other series of sections per animal was stained with standard hematoxylin-eosin (Merck, Darmstadt, Germany) to locate the electrode tips.

\section{Quantitative evaluation of c-Fos immunoreactive cells}

Systematic cell counts were performed for c-Fos immunoreactive (c-Fos-ir) cells in the prelimbic (PrL) and infralimbic (IL) cortices. Photographs of the areas of interest were taken at $4 \mathrm{X}$ magnification using an Olympus DP70 camera connected to an Olympus AX70 bright-field microscope (analySIS; Imaging System, Münster, Germany). Our quantification method was similar to a previously reported method, with minor modifications (Lamprea et al., 2002). Four sections from each animal were selected for quantification. The boundaries of the areas of interest were delineated and measured. The same light intensity and threshold conditions were employed for all sections. The counting of the numbers of c-Fos-ir cells was performed using the conventional image analysis program Image $\mathrm{J}$ (version 1.38, NIH, USA). A cell was counted as c-Fos-ir if its density was $75 \%$ higher than the background density. In addition, artifacts in the sections were excluded from analysis to ensure the accuracy of measurements.

\section{Statistical analysis}

Data are presented as mean \pm S.E.M and were analyzed using Analysis of Variance (ANOVA) repeated measures analysis. Data of the RT task are expressed as follows: MT in sec, RT in sec, and PR as a ratio. Data of c-Fos-ir cell counting are expressed as number of c-Fos-ir cells/105 pixels. A LSD post hoc test was used to analyze group differences in more detail. P-values lower than 0.05 were considered significant. 


\section{References}

Alexander, G.E., Crutcher, M.D., 1990. Functional architecture of basal ganglia circuits: neural substrates of parallel processing. Trends Neurosci. 13, 266-71.

Alexander, G.E., Crutcher, M.D., DeLong, M.R., 1990. Basal ganglia-thalamocortical circuits: parallel substrates for motor, oculomotor, "prefrontal" and "limbic" functions. Prog Brain Res. 85, 119-46.

Anderson, T.R., Hu, B., Iremonger, K., Kiss, Z.H., 2006. Selective attenuation of afferent synaptic transmission as a mechanism of thalamic deep brain stimulation-induced tremor arrest. J Neurosci. 26, 841-50.

Babel, T.B., Warnke, P.C., Ostertag, C.B., 2001. Immediate and long term outcome after infrathalamic and thalamic lesioning for intractable Tourette's syndrome. J Neurol Neurosurg Psychiatry. 70, 666-71.

Baunez, C., Robbins, T., 1997. Bilateral lesions of the subthalamic nucleus induce multiple deficits in an attentional task in rats. The European journal of neuroscience. 9, 2086-2099.

Benabid, A., Pollak, P., Louveau, A., Henry, S., de Rougemont, J., 1987. Combined (thalamotomy and stimulation) stereotactic surgery of the VIM thalamic nucleus for bilateral Parkinson disease. Appl Neurophysiol. 50, 344-346.

Benazzouz, A., Gao, D.M., Ni, Z.G., Piallat, B., Bouali-Benazzouz, R., Benabid, A.L., 2000. Effect of high-frequency stimulation of the subthalamic nucleus on the neuronal activities of the substantia nigra pars reticulata and ventrolateral nucleus of the thalamus in the rat. Neuroscience. 99, 289-95.

Benazzouz, A., Tai, C.H., Meissner, W., Bioulac, B., Bezard, E., Gross, C., 2004. High-frequency stimulation of both zona incerta and subthalamic nucleus induces a similar normalization of basal ganglia metabolic activity in experimental parkinsonism. Faseb J. 18, 528-30.

Blokland, A., 1998. Reaction Time Responding in Rats. Neuroscience \& Biobehavioral Reviews. 22, 847-864.

Chudasama, Y., Muir, J.L., 2001. Visual attention in the rat: a role for the prelimbic cortex and thalamic nuclei? Behav Neurosci. 115, 417-28.

Chudasama, Y., Passetti, F., Rhodes, S.E., Lopian, D., Desai, A., Robbins, T.W., 2003. Dissociable aspects of performance on the 5 -choice serial reaction time task following lesions of the dorsal anterior cingulate, infralimbic and orbitofrontal cortex in the rat: differential effects on selectivity, impulsivity and compulsivity. Behav Brain Res. 146, 105-19.

Dalley, J.W., Fryer, T.D., Brichard, L., Robinson, E.S., Theobald, D.E., Laane, K., Pena, Y., Murphy, E.R., Shah, Y., Probst, K., Abakumova, I., Aigbirhio, F.I., Richards, H.K., Hong, Y., Baron, J.C., Everitt, B.J., Robbins, T.W., 2007. Nucleus accumbens D2/3 receptors predict trait impulsivity and cocaine reinforcement. Science. 315, 1267-70.

DeLong, M.R., 1990. Primate models of movement disorders of basal ganglia origin. Trends Neurosci. 13, 281-5.

Desbonnet, L., Temel, Y., Visser-Vandewalle, V., Blokland, A., Hornikx, V., Steinbusch, H.W., 2004. Premature responding following bilateral stimulation of the rat subthalamic nucleus is amplitude and frequency dependent. Brain Res. 1008, $198-204$.

Evenden, J., 1999a. Impulsivity: a discussion of clinical and experimental findings. J Psychopharmacol. 13,180 - 192.

Evenden, J.L., 1999b. Varieties of impulsivity. Psychopharmacology (Berl). 146, 348 - 361.

Filali, M., Hutchison, W.D., Palter, V.N., Lozano, A.M., Dostrovsky, J.O., 2004. Stimulation-induced inhibition of neuronal firing in human subthalamic nucleus. Exp Brain Res. 156, 274-81.

Gabbott, P.L., Warner, T.A., Jays, P.R., Salway, P., Busby, S.J., 2005. Prefrontal cortex in the rat: projections to subcortical autonomic, motor, and limbic centers. J Comp Neurol. 492, 145-77.

Groenewegen, H.J., 1988. Organization of the afferent connections of the mediodorsal thalamic nucleus in the rat, related to the mediodorsal-prefrontal topography. Neuroscience. 24, 379-431.

Groenewegen, H.J., Berendse, H.W., 1990. Connections of the subthalamic nucleus with ventral striatopallidal parts of the basal ganglia in the rat. J Comp Neurol. 294, 607-22.

Groenewegen, H.J., Galis-de Graaf, Y., Smeets, W.J., 1999. Integration and segregation of limbic cortico-striatal loops at the thalamic level: an experimental tracing study in rats. J Chem Neuroanat. 16, 167-85.

Groenewegen, H.J., Witter, M.P., 2004. Thalamus. The Rat Nevous System, Third Edition. New York, Elsevier. Chapter 17, 407-453.

Heidbreder, C.A., Groenewegen, H.J., 2003. The medial prefrontal cortex in the rat: evidence for a dorso-ventral distinction based upon functional and anatomical characteristics. Neuroscience \& Biobehavioral Reviews. 27, 555-579.

Hubble, J.P., Busenbark, K.L., Wilkinson, S., Penn, R.D., Lyons, K., Koller, W.C., 1996. Deep brain stimulation for essential tremor. Neurology. 46, 1150-3.

Kobayashi, K., Katayama, Y., Kasai, M., Oshima, H., Fukaya, C., Yamamoto, T., 2003. Localization of thalamic cells with tremorfrequency activity in Parkinson's disease and essential tremor. Acta Neurochir Suppl. 87, 137-9.

Kuroda, M., Yokofujita, J., Murakami, K., 1998. An ultrastructural study of the neural circuit between the prefrontal cortex and the mediodorsal nucleus of the thalamus. Prog Neurobiol. 54, 417-58.

Lamprea, M.R., Cardenas, F.P., Vianna, D.M., Castilho, V.M., Cruz-Morales, S.E., Brandao, M.L., 2002. The distribution of fos immuno- 
reactivity in rat brain following freezing and escape responses elicited by electrical stimulation of the inferior colliculus. Brain Res. 950, 186-94.

Loher, T.J., Gutbrod, K., Fravi, N.L., Pohle, T., Burgunder, J.M., Krauss, J.K., 2003. Thalamic stimulation for tremor. Subtle changes in episodic memory are related to stimulation per se and not to a microthalamotomy effect. J Neurol. 250, 707-13.

Lozano, A.M., 2000. Vim thalamic stimulation for tremor. Arch Med Res. 31, 266-9.

Lyons, K.E., Pahwa, R., 2004. Deep brain stimulation and essential tremor. J Clin Neurophysiol. 21, 2-5.

McCracken, C.B., Grace, A.A., 2007. High-Frequency Deep Brain Stimulation of the Nucleus Accumbens Region Suppresses Neuronal Activity and Selectively Modulates Afferent Drive in Rat Orbitofrontal Cortex In Vivo. J. Neurosci. 27, 1260112610.

Mclntyre, C.C., Grill, W.M., Sherman, D.L., Thakor, N.V., 2004a. Cellular effects of deep brain stimulation: model-based analysis of activation and inhibition. J Neurophysiol. 91, 1457-69.

Mclntyre, C.C., Savasta, M., Kerkerian-Le Goff, L., Vitek, J.L., 2004b. Uncovering the mechanism(s) of action of deep brain stimulation: activation, inhibition, or both. Clin Neurophysiol. 115, 1239-48.

Mclntyre, C.C., Butson, C.R., Maks, C.B., Noecker, A.M., 2006. Optimizing deep brain stimulation parameter selection with detailed models of the electrode-tissue interface. Conf Proc IEEE Eng Med Biol Soc. 1, 893-5.

Meissner, W., Leblois, A., Hansel, D., Bioulac, B., Gross, C.E., Benazzouz, A., Boraud, T., 2005. Subthalamic high frequency stimulation resets subthalamic firing and reduces abnormal oscillations. Brain. 128, 2372-82.

Pahwa, R., Lyons, K.E., Wilkinson, S.B., Simpson, R.K., Jr., Ondo, W.G., Tarsy, D., Norregaard, T., Hubble, J.P., Smith, D.A., Hauser, R.A., Jankovic, J., 2006. Long-term evaluation of deep brain stimulation of the thalamus. J Neurosurg. 104, 506-12.

Ray, J.P., Price, J.L., 1992. The organization of the thalamocortical connections of the mediodorsal thalamic nucleus in the rat, related to the ventral forebrain-prefrontal cortex topography. J Comp Neurol. 323, 167-97.

Rubia, K., Smith, A.B., Brammer, M.J., Toone, B., Taylor, E., 2005. Abnormal brain activation during inhibition and error detection in medication-naive adolescents with ADHD. Am J Psychiatry. 162, 1067-75.

Schuurman, P.R., Bosch, D.A., Bossuyt, P.M., Bonsel, G.J., van Someren, E.J., de Bie, R.M., Merkus, M.P., Speelman, J.D., 2000. A comparison of continuous thalamic stimulation and thalamotomy for suppression of severe tremor. N Engl J Med. 342 , 461-8.

Smith, A.B., Taylor, E., Brammer, M., Toone, B., Rubia, K., 2006. Task-specific hypoactivation in prefrontal and temporoparietal brain regions during motor inhibition and task switching in medication-naive children and adolescents with attention deficit hyperactivity disorder. Am J Psychiatry. 163, 1044-51.

Tai, C.H., Boraud, T., Bezard, E., Bioulac, B., Gross, C., Benazzouz, A., 2003. Electrophysiological and metabolic evidence that highfrequency stimulation of the subthalamic nucleus bridles neuronal activity in the subthalamic nucleus and the substantia nigra reticulata. Faseb J. 17, 1820-30.

Temel, Y., Blokland, A., Steinbusch, H.W., Visser-Vandewalle, V., 2005a. The functional role of the subthalamic nucleus in cognitive and limbic circuits. Prog Neurobiol. 76, 393-413.

Temel, Y., Visser-Vandewalle, V., Aendekerk, B., Rutten, B., Tan, S., Scholtissen, B., Schmitz, C., Blokland, A., Steinbusch, H.W., 2005b. Acute and separate modulation of motor and cognitive performance in parkinsonian rats by bilateral stimulation of the subthalamic nucleus. Exp Neurol. 193, 43-52.

Temel, Y., Boothman, L.J., Blokland, A., Magill, P.J., Steinbusch, H.W., Visser-Vandewalle, V., Sharp, T., 2007. Inhibition of 5-HT neuron activity and induction of depressive-like behavior by high-frequency stimulation of the subthalamic nucleus. Proc Natl Acad Sci U S A. 104, 17087-92.

Troster, A.I., Wilkinson, S.B., Fields, J.A., Miyawaki, K., Koller, W.C., 1998. Chronic electrical stimulation of the left ventrointermediate $(\mathrm{Vim})$ thalamic nucleus for the treatment of pharmacotherapy-resistant Parkinson's disease: a differential impact on access to semantic and episodic memory? Brain Cogn. 38, 125-49.

Uylings, H.B., van Eden, C.G., 1990. Qualitative and quantitative comparison of the prefrontal cortex in rat and in primates, including humans. Prog Brain Res. 85, 31-62.

Vertes, R.P., 2004. Differential projections of the infralimbic and prelimbic cortex in the rat. Synapse. 51, 32-58.

Vertes, R.P., 2006. Interactions among the medial prefrontal cortex, hippocampus and midline thalamus in emotional and cognitive processing in the rat. Neuroscience. 142, 1-20.

Wichmann, T., Delong, M.R., 2006. Deep brain stimulation for neurologic and neuropsychiatric disorders. Neuron. 52, $197-204$.

Winstanley, C.A., Eagle, D.M., Robbins, T.W., 2006. Behavioral models of impulsivity in relation to ADHD: Translation between clinical and preclinical studies. Clinical Psychology Review. 26, 379-395. 